\title{
Myxobacterial phytochromes as light-regulated enzymes suitable for XFEL studies
}

\author{
Emina A. Stojkovića ${ }^{\text {, Melissa Carrilloa, Juan Sanchez }}{ }^{a}$, Moraima Noda $^{a}$, Denisse Feliz ${ }^{a}$, \\ Suraj Pandey ${ }^{b}$ and Marius Schmidt ${ }^{b}$ \\ ${ }^{1}$ Department of Biology, Northeastern Illinois University, Chicago, IL 60625, USA, \\ e-stojkovic@neiu.edu \\ 2Department of Physics, University of Wisconsin-Milwaukee, Milwaukee, WI, 53211, USA, \\ smarius@uwm.edu
}

Phytochromes (PHYs) are photoreceptor proteins first discovered in plants, where they control a variety of photomorphogenesis events. PHYs as photochromic proteins can reversibly switch between two distinct states: a red light absorbing (Pr) and a far-red light absorbing (Pfr) form. The discovery of bacteriophytochromes (BphPs) in non-photosynthetic bacteria has opened new frontiers in our understanding of the mechanisms by which these natural photoswitches can control single cell development. BphPs are dimeric proteins that consist of a photosensory core module (PCM) and enzymatic domain, often histidine kinase. The PCM is composed of three domains (PAS, GAF, $\mathrm{PHY}$ ). It holds a covalently bound linear tetrapyrrole (biliverdin, BV) chromophore. Upon absorption of light, the double bond between $B V$ rings $C$ and $D$ isomerizes and switches the protein between $\operatorname{Pr}$ and Pfr states.

The two BphPs from the non-photosynthetic myxobacterium S. aurantiaca, SaBphP1 and SaBphP2, have distinct photochemistry, although they bind the same bilin chromophore and share a large sequence identity. Unlike SaBphP2, the wild-type SaBphP1 lacks a conserved histidine that stabilizes the bilin chromophore in the Pr and Pfr states. We solved the structures of both SaBphP1 and SaBphP2 in wild-type and mutant forms involving substitutions of the conserved His. The wildtype SaBphP2 crystals diffract to a resolution of $1.7 \AA$ at the synchrotron, and microcrystals to about $2.3 \AA$ at the Japanese X-ray Free Electron Laser (XFEL) SACLA, the highest resolution for PCMs so far.

To investigate the structural changes that occur in the phytochrome chromophore, its binding-pocket and the adjacent PHY domain with Time-Resolved Serial Femtosecond X-ray crystallography (TRSFX), we plan to study SaBphP2 using the ultrashort X-ray pulses available at XFELs. Our experiments will not only show structural changes near the chromophore, but also describe signal transduction into the PHY domain which moves several tens of Ångströms after the light stimulus in real time. 


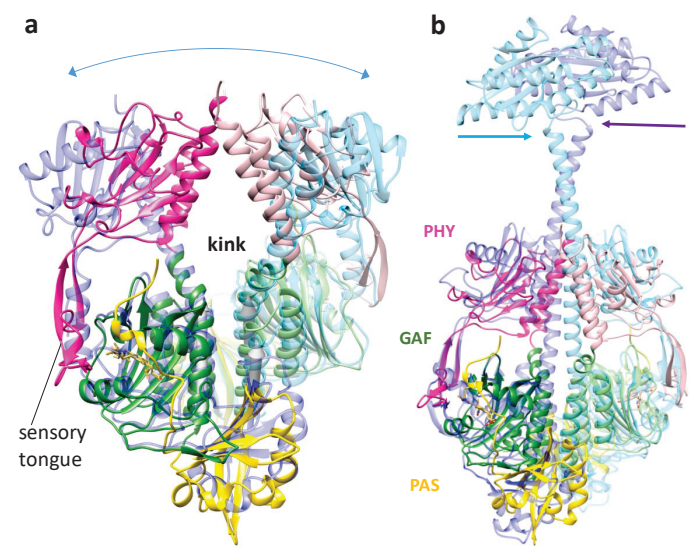

Fig. 1. Structural comparison of SaBphP1-PCM (1) (Pr state in yellow, green, magenta) with related BphPs. Superposition (a) onto the DrBphP-PCM (Pfr) in blue (2) highlighting the displacement of the PHY domains (blue curved arrow) and (b) onto the full length BphP with a di-guanylyl cyclase effector domain from Idiomarina sp. (blue) (3).

\section{$\underline{\text { References }}$}

[1] Woitowich, N. C. et al. (2018). IUCrJ 5: 619-634.

[2] Takala, H. et al. (2014). Nature 509: 245-248.

[3] Gourinchas, G. et al. (2017). Sci. Adv. 3: e1602498. 